\title{
ARTICLE
}

\section{The OPRM1 A118G polymorphism: converging evidence against associations with alcohol sensitivity and consumption}

\author{
M. E. Sloan (D) , T. D. Klepp ${ }^{2}$, J. L. Gowin (D) ${ }^{1}$, J. E. Swan ${ }^{1}$, H. Sun ${ }^{2}$, B. L. Stangl ${ }^{1}$ and V. A. Ramchandani ${ }^{1}$
}

\begin{abstract}
The endogenous opioid system may be involved in the development and maintenance of alcohol use disorder (AUD) and is a target for existing AUD pharmacotherapies. A functional polymorphism of the mu-opioid receptor gene (OPRM1 A118G, rs1799971) may alter the risk of developing AUD. Human laboratory studies have demonstrated that minor allele carriers self-administer more alcohol, show greater sensitivity to alcohol's effects, and exhibit increased alcohol-induced dopamine release. On the other hand, large genome-wide association studies and meta-analyses of candidate gene studies have not found an association between this genotype and alcohol dependence diagnosis. Given this discrepancy, the present study sought to verify whether OPRM1 A118G was associated with alcohol self-administration, subjective response to alcohol, and craving in a sample of 106 social drinkers of European ancestry who completed an intravenous alcohol self-administration session. We found no relationship between OPRM1 rs1799971 genotype and subjective response to alcohol or craving. OPRM1 genotype was not associated with total alcohol exposure or likelihood of attaining a binge-level exposure $(80 \mathrm{mg} \%)$ during the intravenous alcohol self-administration session. Analysis of 90-day Timeline Followback interview data in a larger sample of 965 participants of European ancestry found no relationship between OPRM1 genotype and alcohol consumption in either alcohol dependent or non-dependent participants. These findings suggest that there may not be an association between OPRM1 rs1799971 genotype and alcohol consumption or sensitivity in individuals of European ancestry.
\end{abstract}

Neuropsychopharmacology (2018) 43:1530-1538; https://doi.org/10.1038/s41386-017-0002-8

\section{INTRODUCTION}

The opioid system is centrally involved in hedonic responses [1] and may be implicated in the development and maintenance of alcohol use disorder (AUD) [2]. Alcohol has been shown to trigger endogenous opioid release in rodents and humans [3-5]. This has important treatment implications, as opioid antagonists such as naltrexone reduce relapse to heavy drinking [6]. Given the role of the opioid system in AUD treatment, a functional single nucleotide polymorphism in the mu-opioid receptor gene, OPRM1 A118G (Asn40Asp, rs1799971), has received significant attention. This polymorphism decreases mu-opioid receptor expression [7], resulting in reduced receptor availability in human positron emission tomography studies $[8,9]$. Retrospective analyses of clinical trials suggested that OPRM1 A118G influences naltrexone response [10, $11]$, although a recent well-designed prospective study found no such effect [12]. There is also evidence that OPRM1 A118G affects neurobiological response to alcohol, as healthy male $G$ allele carriers show greater alcohol-induced striatal dopamine release than $A / A$ homozygotes [13]. It is therefore possible that $G$ allele carriers could have altered alcohol sensitivity or alcohol-related behaviors.

Human laboratory studies have largely focused on the relationship between OPRM1 rs1799971 genotype and intermediate phenotypes related to AUD such as subjective response to alcohol. Intermediate phenotypes are thought to be more precise outcome measures than AUD diagnosis and their use is postulated to increase the power to detect the effects of single nucleotide polymorphisms $[14,15]$. Human laboratory studies have provided evidence that $G$ allele carriers have altered alcohol-induced subjective effects [16, 17], increased cue-induced craving [18], and increased intravenous self-administration of ethanol [19,20]. In contrast, a large genome-wide association study (GWAS) and several meta-analyses of candidate gene studies have found no link between OPRM1 rs1799971 genotype and alcohol dependence diagnosis [21-23]. There are several possible reasons for this discrepancy. Existing human laboratory studies have been limited by small sample sizes, which increases the likelihood of detecting spurious associations. Furthermore, these studies may not have adequately accounted for the inclusion of participants from diverse ancestries given that the frequency of OPRM1 rs 1799971 varies by ancestry. On the other hand, the use of alcohol dependence as an outcome in both GWAS and candidate gene studies could also be limiting. Alcohol dependence is a heterogeneous cluster of symptoms and may therefore be less robustly associated with OPRM1 genotype than the more circumscribed outcomes used in human laboratory studies.

The present study aims to clarify the role of OPRM1 rs1799971 in human alcohol consumption. We examined associations between the OPRM1 A118G polymorphism and subjective response to ethanol, craving, and self-administration. To accomplish this, we analyzed data from the largest intravenous alcohol selfadministration sample collected to date $(N=162)$, a substantially larger sample than was used in the original studies demonstrating

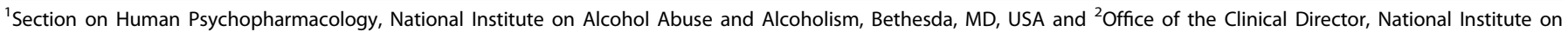
Alcohol Abuse and Alcoholism, Bethesda, MD, USA

Correspondence: V. A. Ramchandani (vijayr@mail.nih.gov)

Received: 20 July 2017 Revised: 28 October 2017 Accepted: 7 December 2017

Published online: 2 February 2018 
associations with alcohol sensitivity $(N=38)$ [17] and intravenous alcohol self-administration $(N=38)$ [19]. To avoid the confounding effects of differential allele distribution across ancestries, we restricted our analyses to participants of European ancestry ( $N=$ 106), the predominant ancestry in our sample. Given the existing human laboratory literature, we hypothesized that $\mathrm{G}$ allele carriers would report greater ethanol-induced subjective effects and higher levels of craving, which would lead to greater self-administer of ethanol. To determine whether $\mathrm{G}$ allele carriers with and without alcohol dependence had higher levels of alcohol consumption outside of the laboratory, we also performed a confirmatory analysis in a sample of nearly a thousand participants of European ancestry who underwent a comprehensive assessment for AUD.

\section{MATERIALS AND METHODS}

Intravenous alcohol self-administration study

Participants. Social drinkers $(N=162)$ between the ages of 21 and 45 participated in intravenous alcohol self-administration sessions at the National Institutes of Health Clinical Center (for detailed demographic information, see Table 1). Ancestry was determined using a genetic panel of ancestry informative markers (AIMs). Participants who were not of European Ancestry (AIMs European ancestry score less than 0.5 ) were excluded without reference to OPRM1 A118G genotype $(N=55)$. One participant was excluded because no information was available about his level of alcohol consumption. The final sample consisted of 106 participants ( $27 \mathrm{G}$ allele carriers). Subjects must have consumed at least 5 drinks on one occasion in their lifetime. Participants were excluded if they met any of the following criteria: (1) non-drinker as per the 90-day Timeline Followback (TLFB) interview [24], (2) lifetime history of alcohol dependence, (3) lifetime history of mood, anxiety, or psychotic disorders, or (4) lifetime history of other substance dependence diagnosis, based on SCID-IV interview [25], (5) recent illicit use of psychoactive substances (e.g., positive urine toxicology screen), (6) history of acute alcoholrelated skin flush [26], (6) regular tobacco use (>20 uses/week, as ascertained during clinical assessment), (7) clinically significant

\begin{tabular}{|c|c|c|c|c|}
\hline \multirow[t]{2}{*}{ Measure } & \multicolumn{2}{|l|}{ Male $(N=56)$} & \multicolumn{2}{|l|}{ Female $(N=50)$} \\
\hline & AA $(N=36)$ & AG/GG $(N=20)$ & AA $(N=43)$ & AG/GG $(N=7)$ \\
\hline \multicolumn{5}{|l|}{ Demographic variables } \\
\hline & Median (IQR) & Median (IQR) & Median (IQR) & Median (IQR) \\
\hline Age & $25.0(5.0)$ & $23.5(5.0)$ & $23.0(6.0)$ & $24.0(2.0)$ \\
\hline AUDIT score ${ }^{a}$ & $6.0(3.0)$ & $6.0(3.5)$ & $5.0(4.5)$ & $6.0(5.0)$ \\
\hline Total drinks ${ }^{\mathrm{b}}$ & $78.0(56.1)$ & $76.5(57.0)$ & $61.0(75.0)$ & $75.0(68.0)$ \\
\hline Average drinks per drinking day ${ }^{b}$ & $3.3(1.7)$ & $3.0(3.0)$ & $2.5(1.7)$ & $2.2(2.1)$ \\
\hline \multirow[t]{2}{*}{ Binge drinking days ${ }^{\mathrm{b}}$} & $3.5(7.8)$ & $2.0(8.8)$ & $4.0(9.0)$ & $5.0(10.0)$ \\
\hline & Mean (SD) & Mean (SD) & Mean (SD) & Mean (SD) \\
\hline Delay discounting ${ }^{a, c}$ & $-5.0(1.8)$ & $-4.7(2.1)$ & $-4.8(1.9)$ & $-4.8(1.3)$ \\
\hline \multirow[t]{2}{*}{ SRE first five ${ }^{d}$} & $4.8(2.3)$ & $4.3(2.1)$ & $3.5(1.2)$ & $4.6(3.0)$ \\
\hline & N (\%) & N (\%) & N (\%) & N (\%) \\
\hline Family history positive & $8(22.2)$ & $5(25.0)$ & $8(18.6)$ & $1(14.3)$ \\
\hline \multicolumn{5}{|l|}{ Outcome variables } \\
\hline & Median (IQR) & Median (IQR) & Median (IQR) & Median (IQR) \\
\hline Feel effects ${ }^{e}$ & $21.0(28.0)$ & $17.0(16.5)$ & $18.0(30.0)$ & $22.0(28.0)$ \\
\hline Like effects ${ }^{f}$ & $56.0(11.8)$ & $53.5(12.0)$ & $52.0(12.0)$ & $58.0(16.0)$ \\
\hline Feel high ${ }^{g}$ & $11.0(29.5)$ & $21.0(28.5)$ & $18.0(31.0)$ & $14.0(24.0)$ \\
\hline Feel intoxicated ${ }^{\mathrm{h}}$ & $16.0(29.0)$ & $15.0(28.5)$ & $18.0(33.0)$ & $22.0(22.0)$ \\
\hline Sedation change $\mathrm{e}^{\mathrm{i}}$ & $2.0(5.0)$ & $2.0(7.8)$ & $1.0(6.0)$ & $0.0(19.0)$ \\
\hline \multirow[t]{2}{*}{ Total alcohol exposure } & $4360.0(6601.4)$ & $5662.5(6268.6)$ & $1514.3(5034.4)$ & $3102.9(6059.7$ \\
\hline & Mean (SD) & Mean (SD) & Mean (SD) & Mean (SD) \\
\hline Want more & $44.0(20.5)$ & $47.5(24.6)$ & $32.9(20.7)$ & $42.7(15.6)$ \\
\hline Post-prime craving ${ }^{\mathrm{m}}$ & $3.3(6.4)$ & $4.2(6.3)$ & $2.4(4.8)$ & $2.1(5.3)$ \\
\hline \multirow[t]{2}{*}{ Stimulation change } & $-0.1(9.8)$ & $1.7(6.5)$ & $-1.6(7.7)$ & $-1.9(6.5)$ \\
\hline & $\mathrm{N}(\%)$ & N (\%) & $\mathrm{N}(\%)$ & $\mathrm{N}(\%)$ \\
\hline Bingers ${ }^{\mathrm{j}, \mathrm{n}}$ & $15(41.7)$ & $9(45.0)$ & $10(23.8)$ & $2(28.6)$ \\
\hline
\end{tabular}

${ }^{a}$ Missing data for some participants: $N=9$ for delay discounting, $N=2$ for AUDIT scores. ${ }^{\mathrm{b}}$ Total drinks, average drinks per drinking day, and binge drinking days over the last 90 days were obtained using the Timeline Followback interview. ${ }^{~}$ Values are reported as the natural logarithm of the discounting constant. ${ }^{d}$ Total score from the Self-Rating of the Effects of Alcohol form (SRE) from the first five drinking occasions. "Measured using the "do you feel any drugs effects" item on the Drug Effects Questionnaire (DEQ) following the priming dose of alcohol. ${ }^{f}$ Measured using the "do you like the effects you are feeling now" item on the DEQ following the priming dose of alcohol. "Measured using the "do you feel high" item on the DEQ following the priming dose of alcohol. "Measured using the "do you feel intoxicated" item on the DEQ following the priming dose of alcohol. 'Biphasic Alcohol Effects Scale (BAES), change measured as BAES stimulant or sedation subscale score following the priming dose of alcohol minus the corresponding baseline subscale score. ${ }^{\mathrm{j}} \mathrm{N}=105$. One female (genotype $=\mathrm{A} / \mathrm{A}$ ) was excluded from alcohol self-administration analyses as her session ended at minute 41.5 due to technical difficulties. ${ }^{\mathrm{K}}$ Total alcohol exposure is defined as the area under the curve of the estimated blood alcohol concentration versus time plot. 'Measured using the "would you like more of what you received, right now" item on the DEQ following the priming dose of alcohol. mPost-prime craving measured as Alcohol Urge Questionnaire (AUQ) score following the priming dose of alcohol minus AUQ score at baseline. ${ }^{\mathrm{n}} \mathrm{A}$ binge during the IV alcohol self-administration session is defined as reaching a software-estimated blood alcohol concentration of $80 \mathrm{mg} \%$ 
Table 2. Characteristics of subjects in the alcohol use disorder assessment sample

\begin{tabular}{|c|c|c|c|c|}
\hline Characteristic & \multicolumn{2}{|c|}{ Non-dependent $(N=406)$} & \multicolumn{2}{|c|}{ Alcohol dependent $(N=559)$} \\
\hline \multicolumn{5}{|l|}{ Demographic variables } \\
\hline & Median (IQR) & Median (IQR) & Median (IQR) & Median (IQR) \\
\hline Age & $27.2(11.1)$ & $26.1(9.3)$ & $43.0(18.0)$ & $46.6(20.8)$ \\
\hline Delay discounting ${ }^{a}$ & $-5.2(2.1)$ & $-4.9(2.4)$ & $-4.5(3.0)$ & $-4.1(2.9)$ \\
\hline Sex (female) ${ }^{b}$ & $137(45.5)$ & $29(27.6)$ & $125(29.8)$ & $45(32.1)$ \\
\hline Family history positive ${ }^{a}$ & $68(26.8)$ & $24(25.5)$ & $259(67.4)$ & $83(65.9)$ \\
\hline Major depressive disorder & $15(5.0)$ & $3(2.9)$ & $70(16.7)$ & $26(18.6)$ \\
\hline Anxiety disorder & $11(3.7)$ & $4(3.8)$ & $142(33.9)$ & $53(37.9)$ \\
\hline Cocaine abuse/dependence & $5(1.7)$ & $0(0.0)$ & $170(40.6)$ & $60(42.9)$ \\
\hline AUDIT score ${ }^{a}$ & $5.0(5.0)$ & $5.0(5.0)$ & $18.0(13.5)$ & $16.0(14.0)$ \\
\hline Total drinks ${ }^{a}$ & $63.0(82.3)$ & $77.0(100.5)$ & $789.8(952.5)$ & $810.0(810.5)$ \\
\hline Average drinks per drinking day ${ }^{a}$ & $2.5(2.0)$ & $2.6(2.3)$ & $12.1(9.7)$ & $11.9(8.0)$ \\
\hline Binge drinking days ${ }^{a}$ & $2.0(9.0)$ & $2.0(12.0)$ & $69.0(54.0)$ & $68.0(48.5)$ \\
\hline
\end{tabular}

alcohol withdrawal (Clinical Institute Withdrawal Assessment for Alcohol Scale Revised score greater than 8) [27], (8) current or chronic medical conditions, including cardiovascular conditions, requiring inpatient treatment or frequent medical visits, or (9) use of medications that may interact with alcohol within the 2 weeks prior to the study. Females were excluded if they were breastfeeding, pregnant, or intended to become pregnant. Although not formal exclusion criteria, none of our participants displayed a heavy alcohol consumption pattern ( $>15$ drinks per week for women or $>20$ drinks per week for men) and only one of our participants met criteria for alcohol abuse.

Genotyping. Genomic DNA was extracted from whole blood using the QIAmp DNA Blood Maxi Kit (Qiagen Hilden, Germany) and run on an Illumina OmniExpress BeadChip array (Illumina San Diego, California, USA). The average genotype reproducibility was more than 0.99994. AIMs [28] were obtained from the array data and ancestral proportions were calculated from extracted markers for all study participants. Ancestry assessment identified six ethnic factors: Africa, Europe, Asia, Far East Asia, Oceania, and Americas.

OPRM1 rs1799971 was genotyped using the assay-on-demand from Applied Biosystems (Foster City, California, USA). The alleles were discriminated by post-polymerase chain reaction plate read on an ABI Prism 7900HT Sequence Detection System. Our sample did not significantly deviate from Hardy-Weinberg equilibrium $\left(x^{2}\right.$ $=0.048, p=0.826$ ).

Laboratory paradigm. Participants completed an intravenous alcohol self-administration session, a paradigm which has shown good test-retest reliability and external validity in prior studies [29, 30]. This paradigm eliminates the large variability in blood alcohol concentration (BAC) associated with oral alcohol intake [31, 32] by bypassing gastrointestinal absorption and first-pass metabolism and using an algorithm that adjusts for age, sex, height, and weight to account for differences in alcohol distribution and elimination [33].
As a result, each infusion increases alcohol levels by a standardized amount, allowing the infusion software to provide continuous estimates of arterial blood alcohol levels that closely track brain alcohol exposure [34] and breathalyzer readouts [35].

Participants were instructed not to drink alcohol in the $48 \mathrm{~h}$ prior to study procedures. Upon arrival, they provided a breathalyzer reading and a urine sample that was tested for illicit drugs (and pregnancy for females). These tests had to be negative to proceed with the study session. After eating a standardized 350 kilocalorie meal, an intravenous catheter was inserted into the antecubital vein in the forearm.

Intravenous alcohol self-administration was conducted using Computer-assisted Alcohol Infusion System software. The session consisted of a 25-min priming phase and a 125-min free access phase. During the first $10 \mathrm{~min}$ of the priming phase, participants were required to push a button four times at 2.5 min intervals. Each button press resulted in an alcohol infusion that raised BACs by $7.5 \mathrm{mg} \%$ in $2.5 \mathrm{~min}$, such that participants achieved a peak concentration of approximately $30 \mathrm{mg} \%$ at minute 10 . During the next $15 \mathrm{~min}$, the button remained inactive while participants experienced the effects of alcohol. Starting at minute 25, participants could self-administer ad libitum, but they had to wait until one infusion was completed before initiating another. Breath alcohol concentration was also obtained via breathalyzer at 15-min intervals to confirm the software-calculated estimates; these readings were entered into the software to provide the model feedback and the infusion rate was automatically adjusted accordingly. Blood alcohol concentration was estimated continuously by the software based on infusion rate and model-estimated metabolism. Software estimates of BAC were used to determine cumulative alcohol exposure, defined as the area under the curve of the estimated BAC versus time plot, and whether a participant reached binge-level exposure, defined as achieving an estimated BAC greater or equal to $80 \mathrm{mg} \%$ [36]. A limit was imposed such that estimated BAC could not exceed $100 \mathrm{mg} \%$ to prevent adverse events due to intoxication. 
Measures. To assess subjective response to alcohol prior to the development of tolerance, we used the first five drinking occasion items on the self-rating of the effects of alcohol form (SRE) [37]. This asks participants how many drinks it took for them to feel different, to feel dizzy, to begin stumbling, and to pass out during the first five drinking occasions. The final score represents the mean of the number of drinks needed to achieve each outcome, with a higher number of drinks indicating a lower level of response to alcohol.

During the intravenous alcohol self-administration session, participants were also assessed for subjective response to alcohol and craving using a series of questionnaires administered at baseline, after receiving the priming dose of alcohol (minute 20), and every $15 \mathrm{~min}$ during the free access phase. Measures included: (1) The Drug Effects Questionnaire (DEQ) [38]: Our DEQ employs a $100 \mathrm{~mm}$ visual analog scale that allows subjects to identify the degree to which they experience five potential outcomes of alcohol intoxication: Feel ("do you feel any drug effects?"), Like ("do you like the effects you are feeling now?"), More ("would you like more of what you received, right now?"), High ("do you feel high?"), and Intoxicated ("do you feel intoxicated?"). (2) The Biphasic Alcohol Effects Scale (BAES): The BAES contains 14 items rated on an 11point Likert scale that collectively measure acute alcohol-induced stimulation and sedation [39]. Seven-item stimulant and sedative subscales were used in our analyses. (3) The Alcohol Urge Questionnaire (AUQ): The AUQ contains eight items rated on a 7point Likert scale to assess alcohol craving [40].

AUD assessment study

Participants. A total of 1836 participants were recruited under three $\mathrm{NIH}$ Institutional Review Board-approved screening and evaluation protocols. Participants were excluded if they were less than 18 , pregnant, or were unable to provide informed consent. Prisoners were also excluded. These protocols serve to extensively phenotype both healthy controls and individuals with AUD in order to (1) collect a common set of measures for all research participants at our institute and (2) determine eligibility to participate in other studies. Thus, all of the alcohol selfadministration participants completed the screening and evaluation protocol prior to being enrolled in the self-administration study. Alcohol self-administration participants were selected from the larger group using the inclusion and exclusion criteria specified in the intravenous alcohol self-administration study section.

To avoid potential confounds from unequally distributed allele frequencies across ancestries, the analysis was limited to individuals of European ancestry $(N=1002)$. Among subjects of European ancestry, those with missing SCID data were also excluded $(N=37)$. The final sample was 965 participants. The participants were divided into those without a current or past diagnosis of alcohol dependence (control group, $N=406$ ) and participants with a current or past diagnosis of alcohol dependence (AD group, $N=559$, Table 2 ).

Measures. All subjects who participated in the intravenous alcohol self-administration study also participated in the screening and evaluation protocol. Thus, measures obtained during this protocol were also used in analyses restricted to the selfadministration sample.

Alcohol consumption was determined using 90-day TLFB interviews, a method of retrospectively estimating daily drinking using a calendar (Sobell et al. 1992). We looked at three outcome measures: total drinks, total number of drinking days (days in which participants consumed at least one drink), and total number of binge days over the 90-day period. We used the National Institute on Alcohol Abuse and Alcoholism's definition of a binge: four or more drinks for women and five or more drinks for men in a given day [36]. TLFB interviews were conducted with $88.0 \%$ of the final sample $(N=849)$. Participants also completed the Alcohol Use Disorder Identification Test (AUDIT), a 10-item questionnaire that assesses problematic alcohol use in the past year [41]. A cutoff score of 8 was used as a marker of risky drinking. Alcohol dependence and abuse diagnoses were determined using the Structured Clinical Interview for DSM-IV axis I disorders (SCID-IV) [25]. Nicotine use was assessed using the Fagerström Test for Nicotine Dependence [42].

Several additional measures obtained during the screening and evaluation visit have been shown to affect intravenous alcohol self-administration [43] and were included as covariates in some analyses. Family history of alcoholism was determined using a two-step process. Participants first completed the Family Tree Questionnaire to identify first-degree relatives and second-degree relatives who may have had alcohol-related problems [44]. Participants subsequently completed the family history assessment plus individual assessment modules of the Semi-Structured Assessment for the Genetics of Alcoholism for all identified relatives to confirm alcohol-related problems [45]. A family history density score was calculated by dividing the number of relatives with alcohol problems by the total number of first-degree relatives and second-degree relatives. A delay discounting task was used to measure behavioral impulsivity [46]. During this task, participants choose between smaller immediate hypothetical rewards or larger hypothetical rewards received after a delay (e.g., $\$ 90$ now or $\$ 100$ in 7 days). The degree of discounting delayed rewards, $k$, is a measure of impulsivity that can be calculated using the equation developed by Mazur [47].

Statistical analysis

Craving and subjective response to alcohol. To ensure that participants experienced alcohol-induced subjective effects and craving following the priming dose of alcohol, we performed Wilcoxon Signed-Rank Tests comparing DEQ and AUQ measures before and after the alcohol prime. To examine whether OPRM1 rs1799971 was associated with craving and subjective response to alcohol, we used linear regression controlled for age and sex. Logtransformations and Box-Cox transformations [48] were employed when necessary in order to meet model assumptions. When the assumptions could not be met, we also analyzed the data using Student's $t$-tests or Mann-Whitney tests split by sex (given that genotype and sex were significantly related in the intravenous self-administration sample, $\left.x^{2}(1)=6.6, \quad p=0.010\right)$. Subjective response to alcohol was also analyzed in a subgroup of risky drinkers (AUDIT score $\geq 8$ ) as this group has been evaluated in

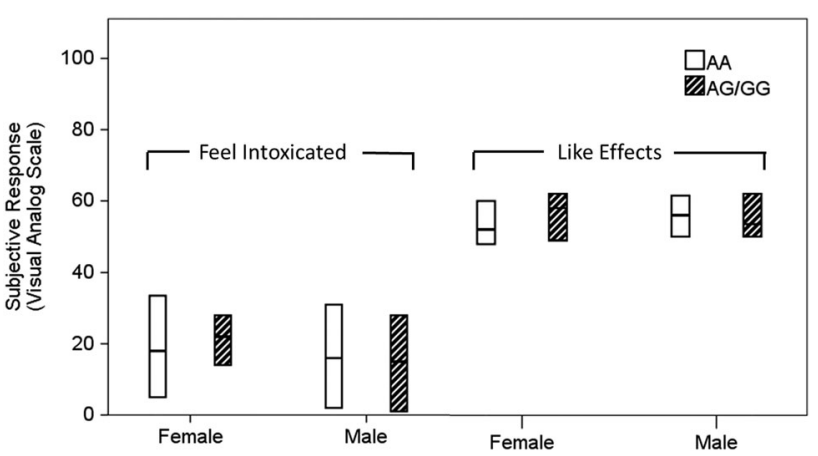

Fig. 1 OPRM1 genotype and alcohol-induced subjective response in the intravenous alcohol self-administration sample. Following the administration of a priming dose of alcohol, subjects reported how intoxicated they felt ("Feel Intoxicated") and how much they liked the effects of alcohol ("Like Effects"). The horizontal line in the middle of each box indicates the median, while the bottom and top borders of the box represent the 25th and 75th percentile values, respectively. No significant differences were observed between genotype groups 
Table 3. Associations between OPRM1 genotype and subjective response to alcohol

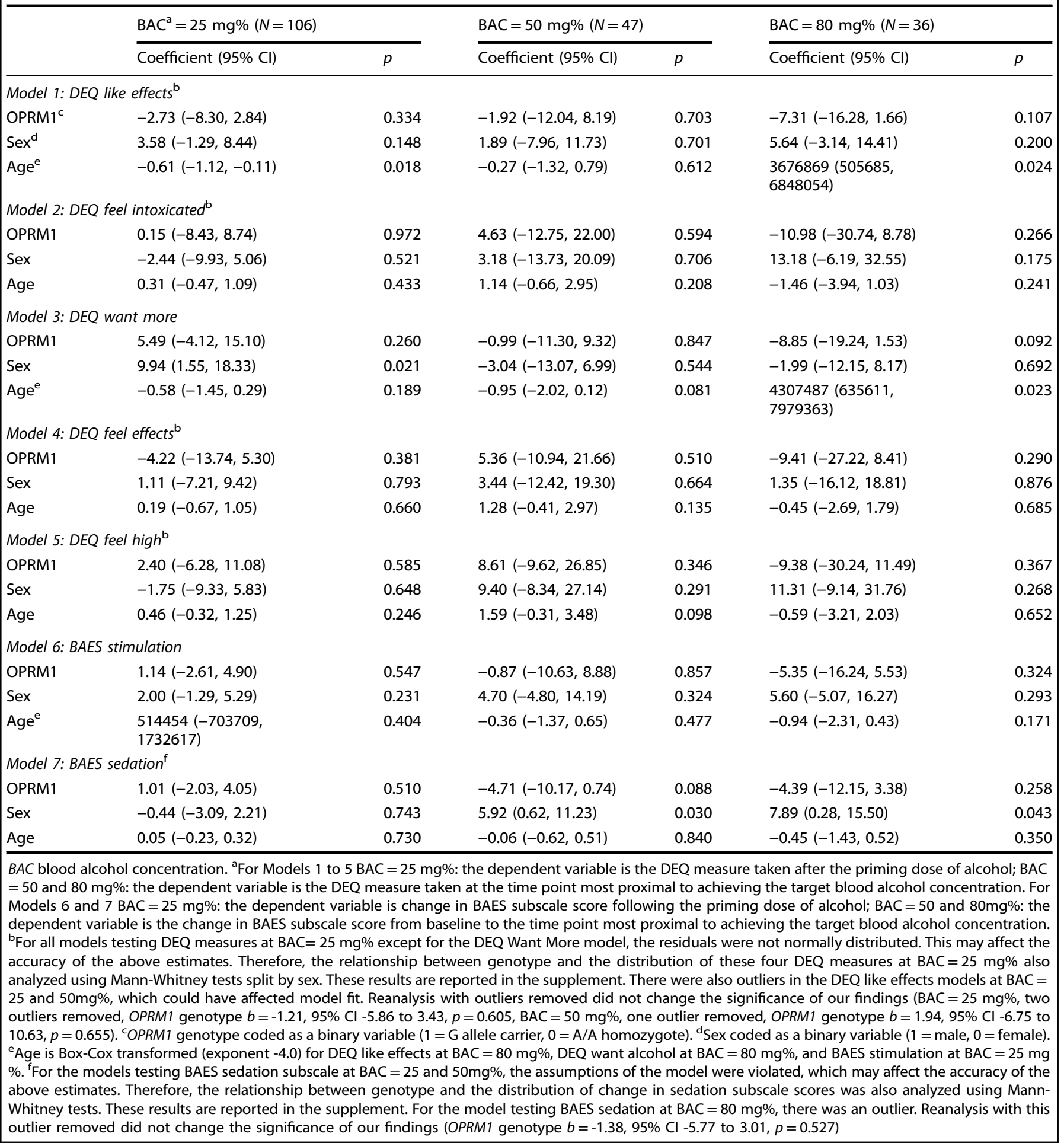

previous studies assessing the effect of OPRM1 A118G on alcohol sensitivity.

Intravenous alcohol self-administration. We plotted Kaplan-Meier Curves split by genotype in males and females to assess the association between genotype and rate of binging throughout the free access phase of the IV alcohol self-administration session. To further examine whether rs 1799971 was a predictor of cumulative alcohol exposure (area under the curve of the breath alcohol concentration versus time plot) and rate of binging, we used linear regression and Cox proportional hazards analysis respectively. For both analyses, the model included genotype, sex, age, delay discounting, and family history of alcohol dependence given that these other measures are associated with rate of consumption [43]. One participant (OPRM1 genotype $=\mathrm{A} / \mathrm{A})$ terminated her intravenous alcohol self-administration session at minute 16.5 of the free access phase as a result of equipment malfunction. Because it is impossible to achieve a binge level of exposure 

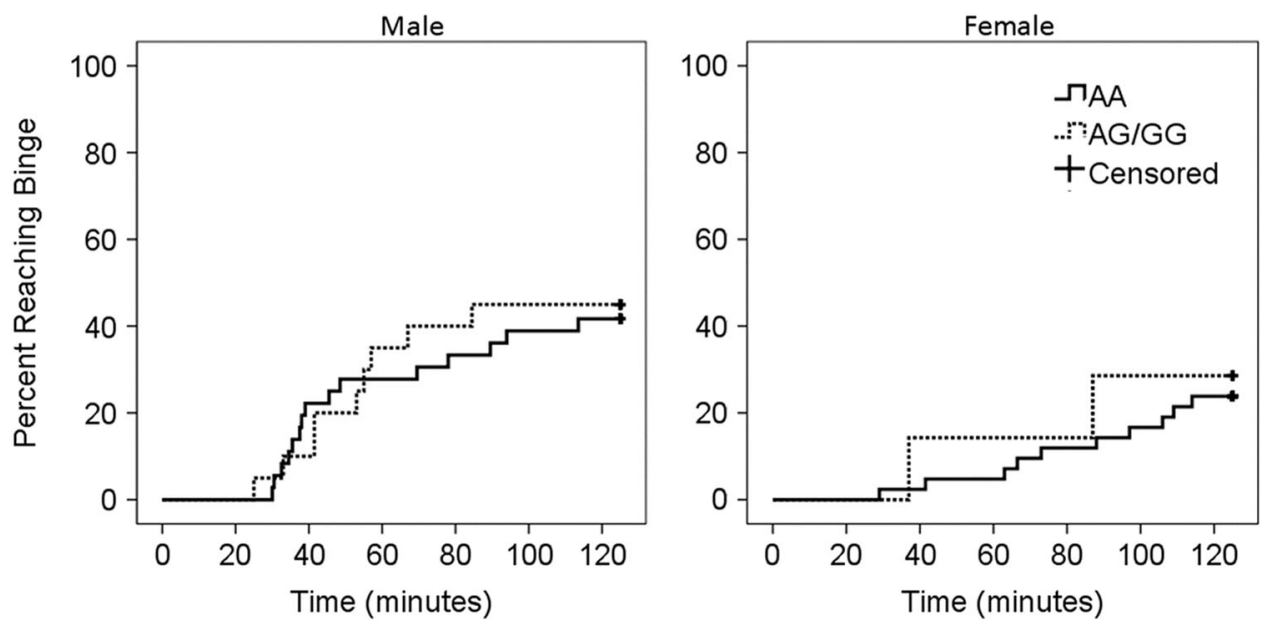

Fig. 2 Kaplan-Meier survival curves examining the effect of OPRM1 genotype on rate of binging in the intravenous alcohol self-administration sample. Panels show the cumulative probability of achieving a binge-level exposure (estimated blood alcohol concentration of $80 \mathrm{mg} \%$ ) during the intravenous alcohol self-administration session in males and females. There were no significant differences between genotype groups

within this timeframe, this participant's data was only used for the subjective response and craving analyses (thus $N=105$ for the alcohol self-administration analyses).

Alcohol consumption. Mann-Whitney tests were used to detect any differences in the distribution of TLFB measures between the two genotype groups in both dependent and non-dependent participants.

Alcohol dependence diagnosis. The relationship between lifetime $A D$ diagnosis and genotype in the AUD assessment sample was analyzed using binary logistic regression while controlling for sex, age, and the most prevalent psychiatric comorbidities in our sample (cocaine and cannabis dependence or abuse, any anxiety disorder, and major depressive disorder).

Given the strong evidence that correcting for multiple comparisons reduces false positive results in candidate gene studies [49], we planned to perform a Benjamini-Hochberg false discovery rate procedure [50] for analyses with multiple outcomes (e.g. DEQ subscales). Uncorrected $p$-values are reported given that none of these analyses reached statistical significance prior to correction.

\section{RESULTS}

Intravenous alcohol self-administration results

OPRM1 genotype and subjective response to alcohol. In the intravenous alcohol self-administration sample, we used the SRE to assess the effect of OPRM1 rs1799971 genotype on subjective response to alcohol during the first five drinking occasions. Linear regression with age (Box-Cox transformed), sex, and genotype as independent variables revealed no effect of genotype on this measure $(b=-0.005,95 \% \mathrm{Cl}-0.879$ to $0.869, p=0.991$, Table S1).

During the intravenous infusion, subjective response was measured following a priming dose of alcohol (median peak BAC attained $=25.38 \mathrm{mg} \%$, interquartile range $=5.92 \mathrm{mg} \%$ ). The priming dose of alcohol led to a significant change in the distribution of all five DEQ measures from baseline $\left(Z_{\mathrm{w}}<-3.0, p<\right.$ 0.001 for all tests). Mann-Whitney tests split for sex revealed no effect of OPRM1 genotype on the distribution of liking alcohol or feeling intoxicated in males (like effects $\mathrm{U}(36,20)=305.0, p=0.345$; feel intoxicated $U(36,20)=338.0, p=0.706$, Fig. 1) or females (like effects $U(43,7)=126.0, p=0.492$; feel intoxicated $U(43,7)=143.0$, $p=0.834)$. Further analyses using the other DEQ items and the BAES stimulant and sedative subscales as dependent variables found no effect of genotype on subjective response following alcohol priming (Table 3 and Supplemental Results). Results remained insignificant when the analyses were restricted to participants with AUDIT score $\geq 8$ (Table S2).

To ensure that a genotype effect would not be observed at higher blood alcohol levels, we examined subjective response to alcohol in subgroups of participants who reached BACs of $50 \mathrm{mg} \%$ $(N=47,14 \mathrm{G}$ allele carriers) and $80 \mathrm{mg} \%(N=36,11 \mathrm{G}$ allele carriers). Subjective response was measured at the time point most proximal to reaching the target BAC. Linear regression models with age, sex, and genotype as independent variables and each of the above DEQ and BAES measures as the dependent variable revealed no effect of genotype on subjective response at either of these BACs (Table 3).

OPRM1 genotype and craving for alcohol. We used the AUQ to examine whether OPRM1 rs1799971 genotype affected baseline alcohol craving and change in craving following the priming dose of alcohol during the intravenous self-administration session. Linear regression with baseline craving (log-transformed) as the dependent variable and age (Box-Cox transformed), sex, and genotype as independent variables found no effect of genotype on baseline craving $(b=-0.034,95 \% \mathrm{Cl}-0.210$ to $0.141, p=0.700$, Table S3). The priming dose of alcohol produced a significant change in craving $\left(Z_{\mathrm{w}}=-5.05, p<0.001\right)$. Student's $t$-tests split for sex revealed that mean alcohol-prime induced change in craving did not differ by genotype in males $(t(54)=-0.462, p=0.646)$ or females $(t(48)=0.152, p=0.880)$.

OPRM1 genotype and alcohol self-administration. We plotted Kaplan-Meier survival curves by OPRM1 rs1799971 genotype and sex to assess the effects of genotype on rate of binging, defined as achieving an estimated BAC $\geq 80 \mathrm{mg} \%$. There was no difference between the two genotype groups (males: log-rank test $=0.044, p$ $=0.834$, females: log-rank test $=0.127, p=0.721$, Fig. 2). We then employed a Cox proportional hazards model to determine whether genotype was associated with rate of binging throughout the session and a linear regression model to determine whether genotype was associated with total alcohol exposure (logtransformed) throughout the session. Age, sex, delay discounting, family history density, and OPRM1 genotype were the independent variables in these models. There was no association between rate of binging throughout the session and genotype (Hazard ratio = $1.030,95 \% \mathrm{Cl} 0.483-2.197, p=0.938$, Table S4) or total alcohol exposure and genotype $(b=0.116,95 \% \mathrm{Cl}-0.347$ to $0.579, p=$ 
A

Participants Without Alcohol Dependence

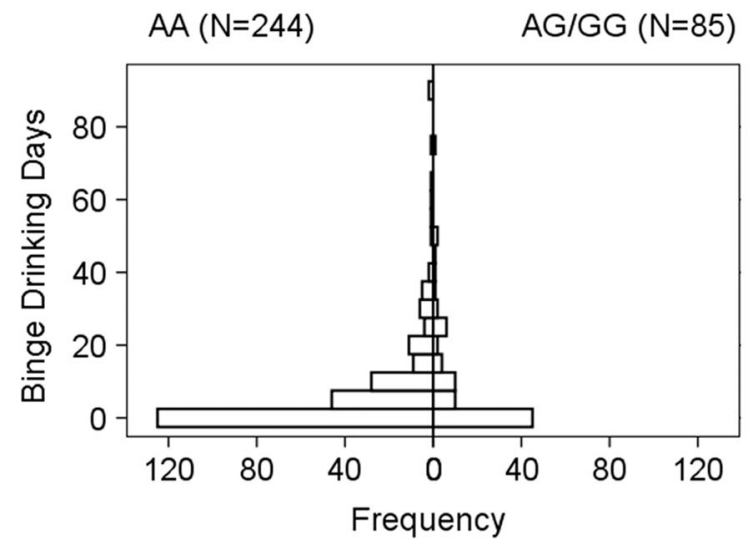

B Participants With Alcohol Dependence

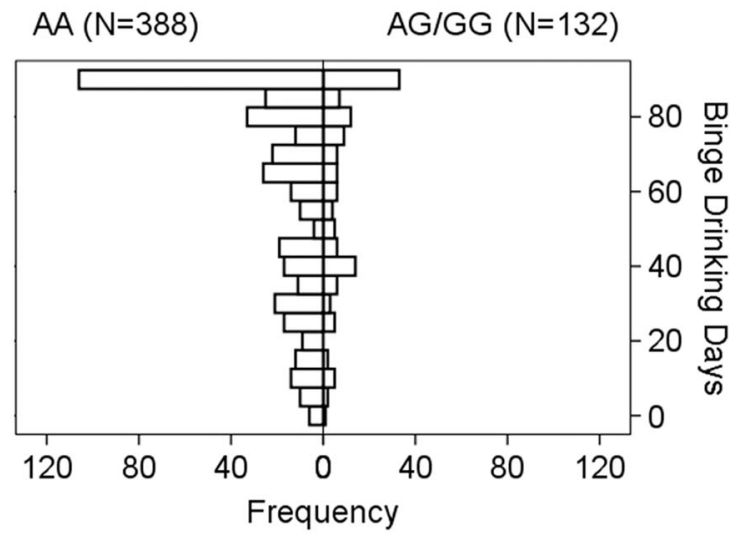

Fig. 3 OPRM1 genotype and binge drinking days in the alcohol use disorder assessment sample. Histograms depict the distribution of binge drinking days in participants without a lifetime history of alcohol dependence (a) and with a lifetime history of alcohol dependence (b). The distributions were not significantly different between genotype groups

0.621 , Table S5). Our findings did not differ when the analysis was restricted to participants with AUDIT score $\geq 8$ (Tables S4 and S5).

AUD assessment sample results

OPRM1 genotype and alcohol consumption. To determine whether there was an association between OPRM1 rs1799971 and real-world alcohol consumption, consumption over the 90 days preceding assessment was analyzed in dependent and non-dependent participants in our AUD assessment sample. There was no effect of genotype on the distribution of total drinks consumed, number of drinking days, or number of binge drinking days in either dependent participants (total drinks $U(388,132)=$ 25297.5, $p=0.835$, drinking days $\mathrm{U}(388,132)=24319.5, p=0.385$, binge drinking days $\mathrm{U}(388,132)=25156.5, p=0.761$, Fig. 3 ) or nondependent participants (total drinks $\mathrm{U}(244,85)=9703.5, p=0.377$, drinking days $U(244,85)=9857.0, p=0.497$, binge drinking days $U$ $(244,85)=10364.5, p=0.994)$. Given that there was a relationship between sex and genotype in non-dependent participants (Table 2), we repeated this analysis split for sex, which did not alter the significance of our findings.

OPRM1 genotype and alcohol dependence diagnosis. OPRM1 rs1799971 minor allele frequencies in alcohol dependent cases and non-dependent controls were 0.132 and 0.140 respectively. Logistic regression was used to investigate the effect of $G$ allele carrier status on the probability of $A D$ diagnosis after controlling for sex and the most common comorbid DSM-IV diagnoses in our sample. Genotype was not a significant predictor of lifetime alcohol dependence when controlling for these variables $(\mathrm{OR}=$ $0.954,95 \% \mathrm{Cl} 0.643-1.417, p=0.816$, Table S6).

\section{DISCUSSION}

Our results provide a strong challenge to the hypothesis that OPRM1 A118G is associated with alcohol consumption. We did not see any differences between genotype groups in either cumulative alcohol exposure or rate of binging during our laboratory paradigm, which provides continuous and accurate estimates of BAC. Furthermore, using a large sample of dependent and nondependent participants, we demonstrated that there is no difference in total alcohol consumption, drinking days, binge drinking days, or likelihood of an alcohol dependence diagnosis between $G$ allele carriers and A/A homozygotes, which accords with a similar analysis of several large Finnish cohorts [51].
In concordance with our consumption analyses, we found that OPRM1 rs1799971 genotype did not affect subjective response to alcohol or craving. We used multiple measures of subjective response. Firstly, we looked at whether genotype affected alcohol sensitivity during the first five drinking occasions, a measure which is subject to recall bias but should not be confounded by alcohol exposure and eventual tolerance. Secondly, using our intravenous alcohol self-administration paradigm, we examined whether genotype affected subjective response following a fixed priming dose of alcohol, a measure which may be confounded by tolerance but is not subject to recall bias. Finally, to determine whether a genotype effect would only be observed at higher levels of alcohol exposure, we examined whether genotype predicted subjective response at blood alcohol levels of 50 and 80 $\mathrm{mg} \%$. These analyses provided convergent evidence against an effect of OPRM1 rs1799971 genotype on subjective response to alcohol. We also found no evidence of differences between genotype groups in baseline or alcohol-induced craving.

Our findings contrast with several prior studies that have reported positive associations between OPRM1 rs1799971 genotype and subjective response to alcohol. These studies have assessed subjective response to alcohol using a variety of measures including the Biphasic Alcohol Effects Scale, the DEQ, and the Profile of Mood States. Many items and subscales derived from these measures are only weakly correlated (for correlations between our subjective response measures following the priming dose of alcohol, see Table S7), and looking at items on a range of scales raises the risk of finding spurious associations due to multiple comparisons. Moreover, OPRM1 rs1799971 genotype has not reliably predicted changes in the same subjective measures across studies. For example, one study found that compared to A/A homozygotes, $G$ allele carriers report greater alcohol-induced sedation but similar alcohol-induced vigor [17], whereas another found that they experience greater alcohol-induced vigor but similar alcohol-induced sedation [16]. Given these limitations and inconsistencies, publication of additional well-powered studies followed by careful metaanalysis of reported findings will be needed to resolve whether an association truly exists between OPRM1 rs1799971 genotype and subjective response to alcohol.

It is possible that OPRM1 A118G may interact with other polymorphisms to influence alcohol sensitivity or consumption. For example, several analyses suggest that OPRM1 A118G's effects on alcohol sensitivity may be influenced by an interaction with a 
40-base pair variable number tandem repeat of the dopamine transporter gene (SLC6A3 or DAT1) [52-54]. In these studies, samples were divided into those homozygous for the 10-repeat allele (A10) and those carrying at least one allele with 9 repeats or less (A9). However, findings to date have been inconsistent. In one study, G allele carriers with two A10 alleles showed steeper alcohol-induced changes in the BAES stimulant subscale than other genotype groups [53]. In contrast, another study found that A/A homozygotes who were A9 carriers demonstrated the greatest alcohol-induced stimulation after consuming a single drink [52], while a third found study found no evidence of an OPRM1 by DAT1 interaction on this subscale [54]. Using the subjective high assessment scale, which predominantly measures negative subjective effects, one study found that $G$ allele carriers with the A9 allele had higher scores compared to the other three genotype groups [54], whereas another did not observe any genotype interaction [52]. These studies had important differences with regards to participant characteristics, dose of alcohol administered, and route of administration. Further studies are therefore necessary to determine whether interactions between OPRM1 A118G and other genetic variants could explain the variable effects on alcohol sensitivity and consumption observed in prior studies.

The effects of OPRM1 A118G genotype in different ancestries remains to be explored. Unlike larger genome wide association studies which are stratified by ancestry, many published human laboratory studies recruit participants from diverse ancestries. This may lead to confounding as minor allele frequency varies substantially by ancestry. Genotype effects on alcohol consumption may also vary by ancestry. For example, there is meta-analytic evidence that Asian but not Caucasian $G$ allele carriers may be at higher risk of alcohol dependence [55]. These possible ancestryspecific effects should be considered in future studies.

There are several limitations to the current study. This is a retrospective analysis of the effects of OPRM1 A118G genotype on consumption and sensitivity; studies that recruit by genotype may produce different results. Nonetheless, analyses were carefully controlled for confounding variables to reduce the possibility that sampling affected our conclusions. Intravenous alcohol administration differs from oral alcohol consumption in that it eliminates alcoholic beverage-related cues such as appearance, smell, and taste. There is evidence that OPRM1 A118G influences cueinduced craving [18], which would not be detected by our paradigm. However, if OPRM1 A118G causes greater cue-induced craving, one would expect that this should translate to greater real-world alcohol consumption, which we did not observe in our AUD assessment sample. Our intravenous self-administration analyses were also limited to social drinkers and the findings may not extend to heavier drinkers. However, we did not observe any genotype effects in exploratory analyses of our participants with AUDIT scores greater or equal to 8. Furthermore, in our AUD assessment sample, we did not find any genotype effects on alcohol consumption in dependent or non-dependent participants. This suggests that there are not differential genotype effects in social versus heavy drinkers. Finally, it has been suggested that $G$ allele carriers may have a slight reduction in overall liability for substance use disorders [23]. Such a small effect would not be detectable in our sample. We were also underpowered to examine whether individuals homozygous for the $G$ allele differed from heterozygotes as there were only $17 \mathrm{G} / \mathrm{G}$ homozygotes in our AUD assessment sample. Larger samples will be required to address this question.

The OPRM1 A118G polymorphism has important physiological consequences including decreased expression of mu-opioid receptors and altered dopaminergic response to alcohol. However, these physiological alterations did not translate into altered subjective response to alcohol, increased craving, or increased alcohol consumption in our sample. This suggests that OPRM1
A118G may not contribute to the development and maintenance of AUD in individuals of European ancestry.

\section{ACKNOWLEDGEMENTS}

Development of the software used for the intravenous alcohol self-administration session was supported by Sean O'Connor, M.D. at the Indiana Alcohol Research Center (NIH P60 AA007611). We are grateful to Julnar Issa, Megan Cooke, Marion Coe, Molly Zametkin, Kristin Corey, Jonathan Westman, Lauren Blau, Courtney Vaughan and the 5-SW day hospital staff for their help in collecting the alcohol selfadministration data. We also thank Dr. Mary Lee, Dr. David T. George, and nurse practitioner Latoya Sewell for their medical supervision of the alcohol selfadministration sessions. Finally, we are grateful to Melanie Schwandt for compiling the alcohol use disorder assessment data set and Ruth Pfeiffer for her analytic suggestions.

\section{AUTHOR CONTRIBUTIONS}

MES and VAR conceptualized the manuscript. MES and TDK conducted the data analysis and drafted the manuscript. JLG assisted with drafting the introduction and with figure preparation. HS genotyped participants for OPRM1 rs1799971. BLS supervised the collection of intravenous alcohol self-administration data. JES assisted with data analysis. All authors revised the manuscript, critically reviewed content, and approved the final version for publication. MES and TDK contributed equally to this manuscript.

\section{FUNDING}

This study was supported by the NIAAA Division of Intramural Clinical and Biological Research (Z1A AA000466).

\section{ADDITIONAL INFORMATION}

Supplementary Information accompanies this paper at https://doi.org/10.1038/ s41386-017-0002-8.

Conflict of interest: The authors declare no conflict of interest.

\section{REFERENCES}

1. Le Merrer J, Becker JA, Befort K, Kieffer BL. Reward processing by the opioid system in the brain. Physiol Rev. 2009;89:1379-412.

2. Nutt DJ. The role of the opioid system in alcohol dependence. J Psychopharmacol. 2014;28:8-22.

3. Jarjour S, Bai L, Gianoulakis C. Effect of acute ethanol administration on the release of opioid peptides from the midbrain including the ventral tegmental area. Alcohol Clin Exp Res. 2009;33:1033-43.

4. Marinelli PW, Quirion R, Gianoulakis C. An in vivo profile of beta-endorphin release in the arcuate nucleus and nucleus accumbens following exposure to stress or alcohol. Neuroscience. 2004;127:777-84.

5. Mitchell JM, O'Neil JP, Janabi M, Marks SM, Jagust WJ, Fields HL. Alcohol consumption induces endogenous opioid release in the human orbitofrontal cortex and nucleus accumbens. Sci Transl Med. 2012;4:116ra116.

6. Jonas DE, Amick HR, Feltner C, Bobashev G, Thomas K, Wines R, et al. Pharmacotherapy for adults with alcohol use disorders in outpatient settings: a systematic review and meta-analysis. JAMA. 2014;311:1889-900.

7. Zhang Y, Wang D, Johnson AD, Papp AC, Sadee W. Allelic expression imbalance of human mu opioid receptor (OPRM1) caused by variant A118G. J Biol Chem. 2005;280:32618-24.

8. Ray R, Ruparel K, Newberg A, Wileyto EP, Loughead JW, Divgi C, et al. Human Mu Opioid Receptor (OPRM1 A118G) polymorphism is associated with brain muopioid receptor binding potential in smokers. Proc Natl Acad Sci USA. 2011;108:9268-73.

9. Weerts EM, McCaul ME, Kuwabara H, Yang $X, X u X$, Dannals RF, et al. Influence of OPRM1 Asn40Asp variant (A118G) on [11C]carfentanil binding potential: preliminary findings in human subjects. Int J Neuropsychopharmacol. 2013;16:47-53.

10. Anton RF, Oroszi G, O'Malley S, Couper D, Swift R, Pettinati H, et al. An evaluation of mu-opioid receptor (OPRM1) as a predictor of naltrexone response in the treatment of alcohol dependence: results from the Combined Pharmacotherapies and Behavioral Interventions for Alcohol Dependence (COMBINE) study. Arch Gen Psychiatry. 2008;65:135-44.

11. Oslin DW, Berrettini W, Kranzler HR, Pettinati H, Gelernter J, Volpicelli JR, et al. A functional polymorphism of the mu-opioid receptor gene is associated with 
naltrexone response in alcohol-dependent patients. Neuropsychopharmacology. 2003;28:1546-52.

12. Oslin DW, Leong SH, Lynch KG, Berrettini W, O'Brien CP, Gordon AJ, et al. Naltrexone vs placebo for the treatment of alcohol dependence: a randomized clinical trial. JAMA Psychiatry. 2015;72:430-7.

13. Ramchandani VA, Umhau J, Pavon FJ, Ruiz-Velasco V, Margas W, Sun H, et al. A genetic determinant of the striatal dopamine response to alcohol in men. Mol Psychiatry. 2011;16:809-17.

14. Enoch MA, Schuckit MA, Johnson BA, Goldman D. Genetics of alcoholism using intermediate phenotypes. Alcohol Clin Exp Res. 2003;27:169-76.

15. Salvatore JE, Gottesman II, Dick DM. Endophenotypes for alcohol use disorder: an update on the field. Curr Addict Rep. 2015;2:76-90.

16. Ray LA, Bujarski S, MacKillop J, Courtney KE, Monti PM, Miotto K. Subjective response to alcohol among alcohol-dependent individuals: effects of the muopioid receptor (OPRM1) gene and alcoholism severity. Alcohol Clin Exp Res. 2013;37:E116-124.

17. Ray LA, Hutchison KE. A polymorphism of the mu-opioid receptor gene (OPRM1) and sensitivity to the effects of alcohol in humans. Alcohol Clin Exp Res. 2004;28:1789-95.

18. van den Wildenberg E, Wiers RW, Dessers J, Janssen RG, Lambrichs EH, Smeets HJ, et al. A functional polymorphism of the mu-opioid receptor gene (OPRM1) influences cue-induced craving for alcohol in male heavy drinkers. Alcohol Clin Exp Res. 2007;31:1-10.

19. Hendershot CS, Claus ED, Ramchandani VA. Associations of OPRM1 A118G and alcohol sensitivity with intravenous alcohol self-administration in young adults. Addict Biol. 2014;21:125-35.

20. Hendershot CS, Wardell JD, McPhee MD, Ramchandani VA. A prospective study of genetic factors, human laboratory phenotypes, and heavy drinking in late adolescence. Addict Biol. 2017;22:1343-54;

21. Gelernter J, Kranzler HR, Sherva R, Almasy L, Koesterer R, Smith $A H$, et al. Genome-wide association study of alcohol dependence:significant findings in African- and European-Americans including novel risk loci. Mol Psychiatry. 2014;19:41-9.

22. Arias A, Feinn R, Kranzler HR. Association of an Asn40Asp (A118G) polymorphism in the mu-opioid receptor gene with substance dependence: a meta-analysis. Drug Alcohol Depend. 2006;83:262-8.

23. Schwantes-An TH, Zhang J, Chen LS, Hartz SM, Culverhouse RC, Chen $X$, et al. Association of the OPRM1 Variantrs1799971 (A118G) with non-specific liability to substance dependence in a collaborative de novo meta-analysis of Europeanancestry cohorts. Behav Genet. 2016;46:151-69.

24. Sobell LC, Sobell MB. Timeline follow-back. In: Measuring alcohol consumption. Berlin, Germany: Springer, 1992. pp 41-72.

25. First MB, Spitzer RL, Gibbon M, Williams JB. Structured clinical interview for DSMIV-TR axis I disorders, Research Version, Patient Edition. New York: Biometrics Research, New York State Psychiatric Institute; 2002.

26. Johnson RC, Nagoshi CT, Schwitters SY, Bowman KS, Ahern FM, Wilson JR. Further investigation of racial/ethnic differences and of familial resemblances in flushing in response to alcohol. Behav Genet. 1984;14:171-8.

27. Sullivan JT, Sykora K, Schneiderman J, Naranjo CA, Sellers EM. Assessment of alcohol withdrawal: the revised clinical institute withdrawal assessment for alcohol scale (CIWA-Ar). Br J Addict. 1989;84:1353-7.

28. Hodgkinson CA, Yuan Q, Xu K, Shen PH, Heinz E, Lobos EA, et al. Addictions biology: haplotype-based analysis for 130 candidate genes on a single array. Alcohol Alcohol. 2008;43:505-15.

29. Junger E, Gan G, Mick I, Seipt C, Markovic A, Sommer C, et al. Adolescent women induce lower blood alcohol levels than men in a laboratory alcohol self-administration experiment. Alcohol Clin Exp Res. 2016;40: 1769-78.

30. Stangl BL, Vatsalya V, Zametkin MR, Cooke ME, Plawecki MH, O'Connor S, et al. Exposure-response relationships during free-access intravenous alcohol selfadministration in nondependent drinkers: influence of alcohol expectancies and impulsivity. Int J Neuropsychopharmacol. 2016; 20:31-9.

31. Friel PN, Baer JS, Logan BK. Variability of ethanol absorption and breath concentrations during a large-scale alcohol administration study. Alcohol Clin Exp Res. 1995;19:1055-60.

32. Ramchandani VA, Plawecki M, Li TK, O'Connor S. Intravenous ethanol infusions can mimic the time course of breath alcohol concentrations following oral alcohol administration in healthy volunteers. Alcohol Clin Exp Res. 2009;33:938-44.
33. Ramchandani VA, Bolane J, Li TK, O'Connor S. A physiologically-based pharmacokinetic (PBPK) model for alcohol facilitates rapid BrAC clamping. Alcohol Clin Exp Res. 1999;23:617-23.

34. Gomez R, Behar KL, Watzl J, Weinzimer SA, Gulanski B, Sanacora G, et al. Intravenous ethanol infusion decreases human cortical gamma-aminobutyric acid and $\mathrm{N}$-acetylaspartate as measured with proton magnetic resonance spectroscopy at 4 tesla. Biol Psychiatry. 2012;71:239-46.

35. Plawecki MH, Zimmermann US, Vitvitskiy V, Doerschuk PC, Crabb D, O'Connor S. Alcohol exposure rate control through physiologically based pharmacokinetic modeling. Alcohol Clin Exp Res. 2012;36:1042-9.

36. NIAAA. NIAAA council approves definition of binge drinking. NIAAA newsletter. 2004; Bethesda: National Institute on Alcohol Abuse and Alcoholism, Vol. 3.

37. Schuckit MA, Smith TL, Tipp JE. The Self-Rating of the Effects of alcohol (SRE) form as a retrospective measure of the risk for alcoholism. Addiction. 1997;92:979-88.

38. Morean ME, de Wit H, King AC, Sofuoglu M, Rueger SY, O'Malley SS. The drug effects questionnaire: psychometric support across three drug types. Psychopharmacology. 2013;227:177-92.

39. Martin CS, Earleywine M, Musty RE, Perrine MW, Swift RM. Development and validation of the Biphasic Alcohol Effects Scale. Alcohol Clin Exp Res. 1993;17:140-6.

40. Bohn MJ, Krahn DD, Staehler BA. Development and initial validation of a measure of drinking urges in abstinent alcoholics. Alcohol Clin Exp Res. 1995;19:600-6.

41. Saunders JB, Aasland OG, Babor TF, de la Fuente JR, Grant M. Development of the alcohol use disorders identification test (AUDIT): WHO collaborative project on early detection of persons with harmful alcohol consumption--II. Addiction. 1993;88:791-804.

42. Heatherton TF, Kozlowski LT, Frecker RC, Fagerstrom KO. The Fagerstrom Test for Nicotine Dependence: a revision of the Fagerstrom Tolerance Questionnaire. Br J Addict. 1991;86:1119-27.

43. Gowin JL, Sloan ME, Stangl BL, Vatsalya V, Ramchandani VA. Vulnerability for alcohol use disorder and rate of alcohol consumption. Am J Psychiatry. 2017;174:1094-101.

44. Vogel-Sprott M, Chipperfield B, Hart DM. Family history of problem drinking among young male social drinkers: reliability of the Family Tree Questionnaire. Drug Alcohol Depend. 1985;16:251-6.

45. Bucholz KK, Cadoret $\mathrm{R}$, Cloninger $\mathrm{CR}$, Dinwiddie $\mathrm{SH}$, Hesselbrock VM, Nurnberger JI Jr., et al. A new, semi-structured psychiatric interview for use in genetic linkage studies: a report on the reliability of the SSAGA. J Stud Alcohol. 1994;55:149-58.

46. Bickel WK, Odum AL, Madden GJ. Impulsivity and cigarette smoking: delay discounting in current, never, and ex-smokers. Psychopharmacology. 1999;146:447-54.

47. Mazur JE. An adjusting procedure for studying delayed reinforcement. In: ML Commons, JE Mazur, JA Nevin \& H Rachlin, editors. Quantitative analyses of behavior, Vol. 5. The effect of delay and of intervening events on reinforcement value. Hillsdale, NJ: Lawrence Erlbaum Associates. 1987. pp 55-73.

48. Box GE, Cox DR. An analysis of transformations. J R Stat Soc Ser B. $1964 ; 26: 211-52$

49. Sullivan PF. Spurious genetic associations. Biol Psychiatry. 2007;61:1121-6.

50. Benjamini Y, Hochberg Y. Controlling the false discovery rate: a practical and powerful approach to multiple testing. J R Stat Soc Ser B. 1995;57:289-300.

51. Rouvinen-Lagerstrom N, Lahti J, Alho H, Kovanen L, Aalto M, Partonen T, et al. mu-Opioid receptor gene (OPRM1) polymorphism A118G: lack of association in Finnish populations with alcohol dependence or alcohol consumption. Alcohol Alcohol. 2013:48:519-25.

52. Anton RF, Voronin KK, Randall PK, Myrick H, Tiffany A. Naltrexone modification of drinking effects in a subacute treatment and bar-lab paradigm: influence of OPRM1 and dopamine transporter (SLC6A3) genes. Alcohol Clin Exp Res. 2012;36:2000-7.

53. Ray LA, Bujarski S, Squeglia LM, Ashenhurst JR, Anton RF. Interactive effects of OPRM1 and DAT1 genetic variation on subjective responses to alcohol. Alcohol Alcohol. 2014;49:261-70.

54. Weerts EM, Wand GS, Maher B, Xu X, Stephens MA, Yang X, et al. Independent and interactive effects of OPRM1 and DAT1 polymorphisms on alcohol consumption and subjective responses in social drinkers. Alcohol Clin Exp Res. 2017;41:1093-104.

55. Chen D, Liu L, Xiao Y, Peng Y, Yang C, Wang Z. Ethnic-specific meta-analyses of association between the OPRM1 A118G polymorphism and alcohol dependence among Asians and Caucasians. Drug Alcohol Depend. 2012;123:1-6. 